\title{
La valeur des études de stabilité des médicaments et de leur publication
}

\author{
par Glen Brown
}

$\mathrm{L}$ e Journal canadien de la pharmacie hospitalière (JCPH) publie depuis longtemps des manuscrits d'études sur la stabilité de préparations extemporanées de médicaments. Les pharmaciens d'établissements du Canada sont chanceux que plusieurs de leurs collègues aient beaucoup travaillé dans ce domaine. Les laboratoires de recherche de Ron Donnelly à l'Hôpital d'Ottawa, de $D^{\text {re }}$ Mary Ensom au British Columbia Children's Hospital et de Scott Walker au Sunnybrook Health Sciences Centre ont fourni grâce à leurs articles dans le JCPH une grande quantité d'information utile qui aide les praticiens à comprendre quelles conditions d'entreposage sont acceptables pour les préparations de médicaments au-delà des produits disponibles sur le marché. Par exemple, le présent numéro contient un article sur la stabilité d'une préparation extemporanée de dompéridone ${ }^{1}$, un type d'études que le comité de rédaction est toujours heureux de publier au sein des pages du Journal. Mais pourquoi?

La fourniture d'une pharmacothérapie selon une formule qui permettra au principe actif d'atteindre le site d'action visé est essentielle au traitement. Or, pour certaines populations de patients des établissements canadiens, cet élément fondamental de la pharmacocinétique ne peut être réalisé à l'aide des produits disponibles sur le marché. Un exemple courant est le scénario clinique dans lequel l'administration par voie orale est souhaitée, mais où le patient est incapable de tolérer le médicament oral disponible sur le marché sous sa forme solide. La situation survient souvent chez les patients très jeunes ou très âgés. Chez les premiers, la posologie demandée peut être trop petite ou la capacité du patient d'avaler est à ce point peu fiable que les formes galéniques sur le marché sont dangereuses ou représentent une option irréaliste ${ }^{2}$. Chez les patients âgés, les cas de dysphagie sont jugés importants, allant de $7 \%$ à $13 \%$, ce qui rend l'emploi des formes pharmaceutiques orales disponibles impossible ou dangereux. De même, les pharmaciens d'hôpitaux canadiens voient souvent des patients atteints de cancers de la bouche, du cou ou de l'estomac qui peuvent rendre impossible la prise des formes pharmaceutiques solides offertes sur le marché ${ }^{\text {. Pour }}$ toutes ces populations, le pharmacien hospitalier pourrait avoir à réaliser des préparations extemporanées de remplacement qui permettront aux patients d'obtenir tous les avantages du traitement. Un autre scénario courant est celui où une maladie de la peau nécessite un traitement topique, car l'administration d'un médicament par voie générale n'est pas souhaitable en raison de possibles interactions avec d'autres médicaments ou maladies. Dans ce cas, le pharmacien pourrait chercher s'il est possible de préparer un produit topique à l'aide d'une forme pharmaceutique d'abord destinée à une administration par voie orale ${ }^{5}$.

Dans l'ensemble des scénarios ci-dessus, il est important de prendre en compte l'efficacité et l'innocuité des préparations extemporanées, tout comme il est souhaitable de s'appuyer sur des données prouvant la stabilité des préparations en question ${ }^{6,7}$. Infostab, un organisme français à but non lucratif, a mis sur pied une base de données présentant les rapports de stabilité publiés pour les préparations extemporanées à administration parentérale et entérale qui pourrait aider les praticiens à préparer un produit nécessaire (www.stabilis.org). Les études de stabilité publiées dans le JCPH apparaissent dans cette base de données.

Le JCPH demeure un forum pour la publication de nouvelles informations sur la stabilité de produits extemporanés. Ces articles aideront les pharmaciens canadiens à se conformer aux recommandations sur la préparation de médicaments de l'Association nationale des organismes de réglementation de la pharmacie $^{8-10}$.

Les études de stabilité soumises au JCPH doivent se fonder sur des méthodes appropriées; ainsi, les lecteurs auront suffisamment confiance aux résultats pour en tirer des conclusions qui leur serviront dans leur propre pratique. La section Renseignements pour les auteurs (Information for Authors) du JCPH précise les descriptions exigées en ce qui concerne le matériel, les conditions d'entreposage, les méthodes 
d'analyse et la reproductibilitée ${ }^{11}$. Des lignes directrices supplémentaires sur les études de stabilité des produits parentéraux ${ }^{12}$ et des antinéoplasiques ${ }^{13}$ sont aussi disponibles. Les auteurs qui soumettent des manuscrits au JCPH doivent se conformer à ces lignes directrices sur la compatibilité $e^{12,13}$ pour ce qui est des préparations médicamenteuses et des voies d'administration précisées dans leurs recherches. Nous accueillons aussi les demandes de renseignements des lecteurs qui souhaiteraient devenir réviseurs d'articles de recherche sur la stabilité de préparations extemporanées. L'expertise de réviseurs permet au Journal de conserver un niveau élevé de qualité pour les publications sur ce sujet.

Les praticiens canadiens continuent de profiter des nouvelles connaissances découvertes grâce aux recherches sur la stabilité des préparations extemporanées et le JCPH continuera à servir de moteur de diffusion pour ces nouvelles informations.

[Traduction par l'éditeur]

\section{Références}

1. Lingertat-Walsh K, Law S, Walker SE. Stability of extemporaneously compounded domperidone $5 \mathrm{mg} / \mathrm{mL}$ suspension in Oral Mix in plastic and glass bottles and plastic syringes. Can J Hosp Pharm. 2018;71(3):165-72.

2. Richey RH, Hughes C, Craig JV, Shah UU, Ford JL, Barker CE, et al. A systematic review of the use of dosage form manipulation to obtain required doses to inform use of manipulation in paediatric practice. Int J Pharm. 2017;518(1-2):155-66.

3. Logrippo S, Ricci G, Sestili M, Cespi M, Ferrara L, Palmieri GF, et al. Oral drug therapy in elderly with dysphagia: between a rock and a hard place! Clin Interv Aging. 2017;12:241-51.

4. Lam MSH. Extemporaneous compounding of oral liquid dosage formulations and alternative drug delivery methods for anticancer drugs. Pharmacotherapy. 2011;31(2):164-92.

5. Chiu HY, Tsai TF. Topical use of systemic drugs in dermatology: a comprehensive review. J Am Acad Dermatol. 2011;65(5):1048.e1-e22.

6. Prankerd RJ. Compounded products - stability studies in hospital pharmacy departments. J Pharm Pract Res. 2009;39(1):5-7.

7. Vigneron J. Stability studies: a scientific mission of the hospital pharmacist. Pharm Technol Hosp Pharm. 2017;2(4):143-4.

8. Modèle de normes relatives à la préparation de produits stériles dangereux en pharmacie. Ottawa $(\mathrm{ON})$ : Association nationale des organismes de réglementation de la pharmacie; 2018. Publié au : http://napra.ca/sites/ default/files/2018-02/Mdl_Stnds_for_Pharmacy_Compounding Hazardous_Sterile_Preparations_Feb2018_FR_FINAL.pdf. Consulté le 10 juin 2018.
9. Modèle de normes relatives à la préparation de produits stériles non dangereux en pharmacie. Ottawa $(\mathrm{ON})$ : Association nationale des organismes de réglementation de la pharmacie; 2018. Publié au : http://napra.ca/sites/ default/files/documents/Mdl_Stnds_Pharmacy_Compounding_Non Hazardous_Sterile_Preparations_April2018_FINAL_FR.pdf. Consulté le 10 juin 2018.

10. Modèle de normes relatives à la préparation de produits non stériles en pharmacie. Ottawa $(\mathrm{ON})$ : Association nationale des organismes de réglementation de la pharmacie; 2018. Publié au : http://napra.ca/sites/default/files/ documents/Mdl_Stnds_Pharmacy_Compounding_Nonsterile_Preparations_ March2018_FINAL_FR.pdf. Consulté le 10 juin 2018.

11. Policy for publication of chemical stability study manuscripts. Can J Hosp Pharm. 1990;43(1):3. Publié au : www.cjhp-online.ca/cshp/templates/ images/Stability_Guidelines.pdf

12. Hecq JD, Bihin B, Jamart J, Galanti L. Criteria for judging the quality of a publication on physicochemical stability of ready to use injectable drugs. Pharm Technol Hosp Pharm. 2017;2(2):79-84.

13. Bardin C, Astier A, Vulto A, Sewell G, Vigneron J, Trittler R, et al. Guidelines for the practical stability studies of anticancer drugs: a European consensus conference. Eur J Hosp Pharm. 2012;19(3):278-85.

Glen Brown, Pharm. D, FCSHP, BCPS (QA), BCCCP, est membre du Service de pharmacie du St Paul's Hospital de Vancouver, en ColombieBritannique. Il est également rédacteur adjoint au Journal canadien de la pharmacie hospitalière.

Intérêts concurrents: Aucun déclaré.

Adresse de correspondance :

Dr Glen Brown

Pharmacy

St Paul's Hospital

1081 Burrard Street

Vancouver (C.-B.) V6Z 1 Y 6

Courriel : gbrown@providencehealth.bc.ca 